\title{
Proposed A Heterogeneous Clustering ALGORITHM TO IMPROVE QOS IN WSN
}

\author{
Mehran Mokhtari ${ }^{1}$ and Masoomeh Tavakoli ${ }^{2}$ \\ ${ }^{1}$ Sama Technical and Vocational Training College, Islamic Azad University, \\ Qaemsharh Branch,Qaemsharh, IRAN \\ ${ }^{2}$ Education Qaemshar, Qaemsharh, IRAN
}

\begin{abstract}
In this article it has presented leach extended hierarchical 3-level clustered heterogeneous and dynamics algorithm. On suggested protocol (LEH3LA) with planning of selected auction cluster head, and alternative cluster head node, problem of delay on processing, processing of selecting members, decrease of expenses, and energy consumption, decrease of sending message, and receiving messages inside the clusters, selecting of cluster heads in large sensor networks were solved. This algorithm uses hierarchical heterogeneous network (3-levels), collective intelligence, and intra-cluster interaction for communications. Also it will solve the problems of sending data in Multi-BS mobile networks, expanding inter-cluster networks, overlap cluster, genesis orphan nodes, boundary change dynamically clusters, using backbone networks, cloud sensor. Using sleep/wake scheduling algorithm or TDMA-schedule alternative cluster head node provides redundancy, and fault tolerance. Local processing in cluster head nodes, and alternative cluster head, intra-cluster and inter-cluster communications such as Multi-HOP cause increase on processing speed, and sending data intra-cluster and inter-cluster. Decrease of overhead network, and increase the load balancing among cluster heads. Using encapsulation of data method, by cluster head nodes, energy consumption decrease during sending data. Also by improving quality of service $(Q o S)$ in CBRP, LEACH, 802.15.4, decrease of energy consumption in sensors, cluster heads and alternative cluster head nodes, cause increase on lift time of sensor networks.
\end{abstract}

\section{KEYWORDS}

Encapsulation, Multi-BS, Multi-HOP, Cloud sensor, Auction cluster head, Alternative cluster head nodes

\section{INTRODUCTION}

Wireless Sensor Networks (WSNs) have been identified as one of the most important technologies of the 21 ST century, and these networks are, new member of wireless networks' family [1]. They have specific features in area. Also, the WSNs are composed of a large number of nodes in a particular area. Each of the nodes are able to collect information on their environment. This informations as follows : temperature, pressure, humidity, noise, lighting, etc. all sensor nodes collect data is send to the base station called sink node [2]. Quality of service in wireless sensor networks is very different from the traditional networks [3]. Quality of Service parameters (maximum acceptable delay, packet loss, and, etc.) depending on the type of application is important. Thus, pay attention to the standards for the design and choice of clustering methods is important. For example, qos in RFC 2386 as a set of service requirements be considered . [4]. QoS should be considered when a packet is sent from source to destination. In this regard, the quality of service is presented as follows :

The internet is guaranteed to provide a set of properties of appropriate services for users, or endto-end applications based on the delay, jitter, bandwidth and packet loss. Guarantee Internet for provide a set of services for users or applications, such as end-to-end in terms of delay, jitter,

DOI: $10.5121 /$ ijcnc.2016.8402 
bandwidth and packet loss. There are two approaches to service quality that can be shown by a simple model . [2][5]. QoS parameters of WSNs may have different types, which depend on network application. Different types of QoS parameters there is in wireless sensor networks, that will depend on the type of application. Some quality assessment parameters include: The whole network covering, the optimal number of active nodes in the network, the accuracy of the observation (measurement accuracy of the sensor), the accuracy of the data transfer, data transfer in the shortest possible time, reliability, network lifetime and energy consumption in the networks. [6]. As defined in [7,8], Quality of Service is a set of service requirements to be met by the network while transporting a flow. "Here a flow is" a packet stream from source to a destination (unicast or multicast) with an associated (QoS) [7]. In other words, QoS is a measurable level of service delivered to network users, which can be characterized by packet loss probability, available bandwidth, end-to-end delay, etc. In this section, first, we have discussed about the QoS requirements \& Challenges in WSNs followed by the major existing approaches for supporting QoS in WSNs. The requirement of QoS in WSNs can be specified from two perspectives [9]. These are application specific QoS and Network QoS. As discussed in Section 1, QoS parameters in WSNs may vary, depending on the application domain. Some of the application specific QoS parameters are data accuracy, aggregation delay, fault tolerance, coverage [9], optimum number of active sensors [4] etc. The application demands certain requirements of the deployment of sensors which are directly related to the quality of application.

From the network perspective, it has been considered as how to provide QoS constrained sensor data while optimally utilizing sensor resources. Every class of application has some common requirements in network. The network is concerned with how to transmit the sensed data from the sensor field to the sink node fulfilling the required QoS. There are three data delivery models in sensor network [2]. These are event driven, query driven and continuous. The event driven application in WSNs is mostly delay tolerant, interactive and non End-to-End. The sensors detect the occurrence of certain events and to take action accordingly. In one side of the application there is a sink node and on the other side a group of sensor nodes which are affected by certain events [5]. The query driven application WSNs are interactive, query based, delay tolerant, mission critical and non end-to-end. The queries are generated by the sink node on demand and sent to sensor nodes inquiring occurrence of certain events. In traditional data network, QoS defines certain parameters such as packet loss, delay, jitter, bandwidth, and, etc. However, the QoS requirements in WSNs such as data accuracy, aggregation delay, coverage, fault tolerance and network lifetime, and, etc. In general we can say that energy in wireless sensor network (WSN) based on clustering are used the following 4 case (items):

1. Communication.

2. Processing, or calculation of intra-cluster.

3. Sensing data.

4. Actuating.

As we know cluster heads (CH's) have different responsibilities such as:

Data aggregation, Data fusion, Processing of collective data from the other intra-cluster sensors locally, Sending collective data, Scheduling of intra-cluster, and specified sleep mode for sensors of intra-clusters, decrease number of sending messages, or prevention of accumulate messages, Load balancing, Reduce over load, Reducing routing table. Reducing delay on contact with BS. At first protocols CBRP, LEACH, and 802.15.4, all the works were reviewed. Then the following steps are given: 
International Journal of Computer Networks \& Communications (IJCNC) Vol.8, No.4, July 2016

The basic assumptions of the proposed algorithm, General description and application of the proposed algorithm, Phase cluster head selection algorithm auction, Cluster formation phase selection algorithm, using scheduling phase algorithm, and sleep/wake, calculation amount of energy during sending data, process of intra-cluster locally, sending the collected data by cluster head for base station Multi-HOP and collaborative, using backbone sensor network organized of head clusters, and solution problem of Multi-BS network, using three level heterogeneous clustering hierarchical wireless sensor network, and using collective intelligence, group interactions of intra-cluster, using alternative group of cluster nodes on each cycle to cluster head selection, simulation, analysis of quality service parameter, and conclution. This document describes, and is written to conform to, author guidelines for the journals of AIRCC series. It is prepared in Microsoft Word as a .doc document. Although other means of preparation are acceptable, final, camera-ready versions must conform to this layout. Microsoft Word terminology is used where appropriate in this document. Although formatting instructions may often appear daunting, the simplest approach is to use this template and insert headings and text into it as appropriate.

\section{OVERVIEW OF 802.15.4, LEACH, AND CBRP Protocols}

IEEE 802.15.4 is the emerging next generation standard designed for low-rate wireless personal area networks (LR-WPAN) [11]. IEEE 802.15.4 protocol is a standard for low rate -wireless personal area network (LR-PAN). It consists of low cost, less complex, low energy consumption, star topology, and also peer-to-peer is another particular things of this standard [11]. Physical layer of this standard is $868 / 915 \mathrm{MH}$, or almost $2.4 \mathrm{GH}$. MAC layer uses from CSMA-CA mechanism. It is also designed for WSNs applications, that need low range communication with maximum battery life time, it allows star topology, and point to point topology to be formed between networks [12] [5]. Zig-Bee technology are created satisfy market need for its low price with low potential, and low rate for WSNs. This technology is formed based on IEEE 802.15.4 standard. It defines layers of PHY, and MAC to transfer data with low rate in (LR-WPANS) low rate wireless personal area network [12]. IEEE 802.15.4 standard is also known as of wireless personal network with low rate(LR-WPAN). It is wireless communication network with low range, and less complicated, are used for application programs, with limit potential, and it has been designed for fixed necessity, efficiency. On the other hand 802.15.4 standard protocol doesn't privately defines for sensor networks, but it is not the same for WSNs. This standard is part of WPAN family, usually has a 10 meters of Personal Operating Space (POS). The sensor network community has a strong background to attract in the use of these protocols for sensor networks.Recent studies have shown IEEE 802.15.4 MAC protocol in terms of efficiency, reliability, and energy (if otherwise proper parameter setting) may be severely limited. [20] Hence, to adjust the parameter optimization of the reliability and minimum energy consumption Some solutions have been proposed. IEEE 802.15.4 is a standard for low-rate, low-power, and low-cost Personal Area Networks (PANs). The IEEE 802.15.4 physical layer offers a total of 27 channels, one in the $868 \mathrm{MHz}$ band, ten in the $15 \mathrm{MHz}$ band, and sixteen in the $2.4 \mathrm{GHz}$ band .[20] WSNs consists of Base Stations (BS) and several nodes which are unattended and have limited resource and they can work until the energy exists inside them [9]. Low energy adaptive clustering hierarchical (LEACH) is the first network protocol that uses hierarchical routing for WSNs to increase the life time of network . LEACH is a cluster based protocol. Performance of LEACH protocol evaluated in NS2 simulator by following [9] : 1) throughput ; 2) energy of cluster heads; 3) packet delivery ratio; 4) end-to-end delay. Among communication protocols presented, LEACH protocols are particular importance to researchers for the following reason. 
First, a cluster networks are formed randomly, adaptively, and self-configured. On the other hand Cluster Based Routing Protocol (CBRP) is a robust/scalable routing protocol for Mobile Ad-hoc Networks (MANETs) superior to the existing. Since CBRP is a robust and scalable routing protocol for Ad-hoc, CBRP is used as a routing protocol in our simulations. In this routing protocol all nodes divided into some clusters. Any cluster node connects to other clusters by cluster head via inter-links between them. To reduce energy consumption in this type of network we put all idle member nodes except cluster heads and gateways nodes to sleep mode. Our experiments show that the energy consumption is significantly reduced but to have the maximum saving energy we must pay its expenses by packet delivery delay. CBRP is a robust/scalable routing protocol for MANETs and superior to the existing methods [9] (e.g. the overhead of it is less than and throughput of it is more than of AODV) [3]. CBRP is a routing protocol designed for medium to large mobile ad-hoc networks. The protocol divides the nodes of the ad-hoc network into a number of overlapping or disjoint 2-HOP diameter clusters in a distributed manner. Each cluster chooses a head to retain cluster membership in-formation. The algorithm is a variation of the "lowest ID" cluster algorithm. The node with a lowest ID among its neighbors is elected as a Cluster Head $(\mathrm{CH})$. Each node maintains a neighbor table and a cluster adjacency table. Neighbor table is a conceptual data structure that it employs for link status sensing and cluster formation. Cluster adjacency table keeps information about adjacent clusters for adjacent cluster discovery. These tables are updated by the periodic Hello Messages (HM) [9].

\section{RELATED WORK}

A WSNs contains hundreds or thousands of these sensor nodes. These sensor nodes are able to communicate with each other or communicate directly with the base station .Sensor nodes can be used to collect information from the environment, locally process this data and transmit the sensed data back to the user. Basically, each sensor node comprises sensing, processing, transmission, mobilize, position finding system, and power units. These nodes collect and transmit the information [12]. On reference [4], QoS are in 3 different groups: quality of end-toend service(achieve the speed or accuracy end-to-end transmission) reliability assurance (ensure packets reach destination) an depends on the application (number of active nodes). On reference [2] two parameters of data transfer and energy consumption of a communication channel in sensor network is considered as a base for quality service. On reference [13] multiple routing methods are introduced as a technique to improve QoS in WSNs. Multiple routing methods by creating several multiple routes between the source and destination of nodes improve criteria such as reliability, power consumption, delay, throughput and bandwidth. On reference [14] analysis of IEEE 802.15.4 Zig-Bee performance for star topology with the traffic scenarios CBR, FTP with simulation using NS. Packet delivery rate is 100 percents on a star topology with CBR traffic compared to the other traffics. Because with CBR traffic there is no acknowledgement for control. Lost packets are less on CBR, because it provides constant bit rate during the simulation on general factor of traffic load on CBR traffic is larger. In this article [14] to improve the performance of star topology for programming of simulation scenarios like sensors in WSNs to a control central nodes, monitoring of systems in the future are suggested. On reference [15] it is shown: One of the important quality service evaluation is the number of the sender, or active nodes in the network. According to increase of sensors, by activate of a number of sensors, and disactivate the rest of them, same time improving the QoS requirements, increase the lifetime of the network. Active or deactive method of sensors have a direct effect over the lifetime of those, and network. Because giving a method that use equal energy of all nodes is very effective to increase network lifetime. This study presents a method based on sensors energy to control service quality. Comparing this method with similar methods is shown that using this method not only QoS increased, but was also increased life time of the network very much. On reference [16] 
several QoS-aware protocols are introduced. These protocol minimize the band-width usage and delay. Also choose the routes with minimum expenses to communication, assure to transfer packet. Thus the service quality is very important according to the kind of application in WSNs is very different, comparing to the old networks. Therefore research on efficiency of standard protocols, and base for satisfying service quality are necessary to improve performance of the network. An improved and more efficient protocol for WSNs in order to provide QoS and improve service parameters can enhance the performance and efficiency of the network. In this context, improving service quality, schedule, cost and time spend by type of program in WSNs as the optimal can increase the lifetime of network. Since a variety of applications in WSNs need to provide different quality parameters such as delay, reliability, amount of bandwidth, etc. Design of a protocol due to the problems caused by the unique characteristics of WSNs such as the limited bandwidth, unreliable communication, vulnerability of nodes and more. Requirements a reconsidered essential for QoS in networks. The result of this research can provide guidance and direction to research and solve problems related to QoS requirements, and help to achieve the required QoS in WSNs. In this article [6], QoS parameters were studied in detail to evaluate the performance of three protocols. To do it, first three protocols 802.15.4, LEACH and CBRP were separately simulated in the NS simulator. Then we analyzed the simulation output. For evaluation of the performance of CBRP protocol, it used the NS 2.34. Simulation was done with a constant number of nodes in WSNs. The protocol used for the three protocols was Ad-hoc on demand distance vector. QoS parameters were analyzed with following titles: average delay, throughput, energy consumption, number of packets lost, packet delivery ratio, normalization network and mean HOP. We were able to compare the protocol due to changes of the parameters of QoS. Also, the simulation results of CBRP algorithm were compared with other two protocols.

Strengths and weaknesses of QoS parameters were known over the three protocols. Thus, by knowing the status of each one (each protocol) we can solve the weaknesses. About the packet delivery rate, LEACH protocol has a weakness. CBRP protocol has strengths. The 802.15.4 protocol has a fixed rate. At last, by analyzing all parameters of quality services, we got this conclusion that CBRP, route protocol for supplying service qualities is better and more efficient. Therefore it is more efficient and suitable to supply quality service. It has developed QoS more than the other two protocols. Also, using extensive simulation, we demonstrate the robustness of models and expand the initial work done in [1] [7] [8] on the above performance parameters .

These include the assessment between multi-constrained QoS multipath routing in WSNs and the impact of AODV Protocol on the intensity of the network performance. Also, it shows the effect of the AODV protocol which ensures the efficiency [6].

In this paper [18] consider a case where variable power levels could be utilized for intra cluster communications. In this condition MinPwri is the minimum power level required for node $i$ $(1<i<M)$ so that VI is able to communicate with a cluster head, such as U. M denotes the number of nodes in the cluster range. Average Minimum Reachability Power (AMRP) is considered as average needed power level for all $M$ nodes in the cluster range for reaching $u$. [18]

$$
\mathrm{AMRP}=\frac{\sum_{\mathrm{i}=1}^{\mathrm{M}} \mathrm{MinPwr}_{\mathrm{i}}}{\mathrm{M}}(\text { Equ. 1) }[18]
$$

If each node is allowed to choose proper power level to access its cluster head, AMRP provides an acceptable estimation of communication cost. If a node is cluster head, AMRP of node is a measure of required energy in cluster communications. Clustering algorithm is executed each $T p c$ + Tno seconds to select new cluster heads. In each node clustering process needs a few steps. The 
International Journal of Computer Networks \& Communications (IJCNC) Vol.8, No.4, July 2016

number of steps is represented by Niter. Each step lasts $T c$ (enough time for messages to reach from each neighbor). The probability of being cluster head, $C_{\text {prob}}$, equals to: [18]

$$
\mathrm{CH}_{\text {prob }}=\mathrm{C}_{\text {prob }} * \frac{\mathrm{E}_{\text {residual }}}{\text { Emax }}(\text { Equ. 2) }
$$

\section{The Basic Assumptions Of The Proposed Hybrid Clustering AlgorithM}

In this algorithm (leach extended hierarchical 3-level : LEH3L) we consider coverage area(sensor field) as a geometric shape such: Square, Circle, or etc. For example in the square in dimension $1000 \mathrm{~m} * 1000 \mathrm{~m}$ we distribute randomly 100 sensor nodes by a device $(\mathrm{M}=100)$. We assume that the maximum energy of each sensor node on a best condition (full charge) be equal to $50 \mathrm{j}$. Also, We assume that the minimum energy of each sensor node on a lowest condition (Free of charge) be equal to $0 \mathrm{j}$. In this case, the node life to ends. ( $0 \mathrm{j}$ condition means the end of life time of one node, and it is called death time). In this stage we should give a number to each one of the sensor of node randomly in internal [ $\mathrm{c}$ to $50 \mathrm{j}$ ] that $\mathrm{c}$ is a little higher than zero, because being zero, the remaining of node energy means completely empty battery, in fact it means the death of node.

Nodes and cluster heads assumed constant. As a result it is not considered, mobility. Nodes which have the highest energy, first, at the beginning of algorithm (starting simulation) or have the highest remaining energy after each round, called the cluster head or $\mathrm{CH}$. Therefore, according to the given description it is clear that suggested algorithm acts based on the remaining node energy on each cycle. Using this criteria we can calculate the usage energy average by each node to send data from source node to destination nodes. It can be calculated as shown below:

$$
\mathrm{E}_{\mathrm{A}}=\frac{\sum_{\mathrm{I}=1}^{\mathrm{M}}\left(\mathrm{e}_{\mathrm{i}, \text { init }}-\mathrm{e}_{\mathrm{i}, \mathrm{res})}\right.}{\mathrm{M} \sum_{\mathrm{I}=1}^{\mathrm{S}} \text { dataN }_{\mathrm{j}}}(\text { Equ. 3) }
$$

In this formula parameter $\mathrm{M}$ indicates number of nodes in network. Parameters init, $\mathrm{e}_{\mathbf{i}}$, $\mathrm{e}_{\mathrm{i}}$,res respectively show amount of initial energy, and remaining energy in ith node. Parameter $S$ include number of destination nodes, and parameter $\mathrm{N}_{\mathrm{j}}$ also determines the amount of received data by jth destination node. The result of simulation of most routing protocols indicate, that if the number of the nodes in network increase, rationaly ability of multi routing to indentify more routing will increase. So amount of sending traffic by each node decrease, and the average consumption energy on each node will also decrease.

Heterogeneous WSNs may contain two, three, or multi types of nodes with respect to their energy levels and termed as two, three, or multi-level heterogeneous WSNs, respectively [19]. Clustering is a key technique to improve the network lifetime, reduce the energy consumption and increase the scalability of the sensor network. In this paper, we study the impact of heterogeneity of the nodes to the performance of WSN. This paper surveys the hybrid clustering algorithm for heterogeneous WSN.

\section{General Description Of The Proposed Algorithm}

Stages of implementation of the proposed algorithm are as follows:

1. This program at first makes structure, and list of tables.

2. Creates network nodes. 
International Journal of Computer Networks \& Communications (IJCNC) Vol.8, No.4, July 2016

3. Calculate number of clusters according to the area network.

4. According to clusters selects cluster heads (identifies cluster heads according to energy of nodes or highest router ID) and picks the nodes in clusters (arrange the nodes in clusters) On each cluster node selects the alternative cluster head to increase speed of network.

5. Sets the data center station (such as nodes are close to it picks as a single cluster head) and specifies the interface to the cluster head.

6. Routing table fills up in inter-cluster \& intra-cluster It will select a boarder line among each cluster node and other clusters.

7. Each cluster head sends a signal for existing nodes in cluster to identify itself by cluster nodes.

8. Then problem of overlapping of node by two or more clusters are solved, orphaned or unused nodes also will be identified.

9. So routing algorithms intra-cluster by auts and grid algorithm will be implemented. Also intracluster processing will be done locally. Least energy 0\%, maximum energy $100 \%$ are defined.

The nodes that are closer to base station they send data directly. In this program the method to identify cluster member by cluster head is shown to as bellow: Cluster head sends a signal to nodes around it, if the cluster number of those were the same as cluster number of cluster head, it will save it as a reserve member. In this program amount of energy during the sending data will be calculated. It decrease the energy of involve nodes. When cluster heads data send to cluster head, or nodes alternative cluster neighbor. It uses compress algorithm to form it like a compressed and then sends it. This function will help to reduce amount of energy in network.

Also this program before will be sent two things as of high header, taylor (for data security) are added to data, and then will send. Next, we try to describe the processing of algorithm step by step from the beginning of simulation until completely the end of it.

\section{Auction Cluster Head Selection Algorithm Phase}

In proposed algorithm using ideas of selected cluster head algorithm in routing protocol (OSPF) two stages are used by one method selecting cluster head. In fact selecting cluster head, or cluster heads by two stages auction as following.

A. Stage 1: First each one of the existance sensors in network, give a random number like $\mathrm{P}$ (in this algorithm $0 \mathrm{j}<=\mathrm{p}<=50 \mathrm{j}$ ) that is equal to priority of those to accept cluster head's role. Therefore each one of the sensors that have higher number of priority, can be chosen as a cluster head.

B. Second step: When sensors nodes in first step have equal priority number in clusters or after forming clusters, it should be compete with each other based on greater router ID's parameter. And sensor that has greater router ID, be selected as of cluster head node. In fact router ID defines as a greatest (largest) IP address of each one of the sensors of wireless sensor in network. Therefore when it is time to distribute the sensors in environment (sensor field) we assign a IP address to each one of the sensors before. As this IP addresses be unique (without any conflict) to the sensors by one access point that protocol DHCP is active on it, scopes assign from a range of address. Therefore, the assumption made and the realization of the stated (using of IP address for each of the sensors), each one the clusters should be similar to a subnet (independent), and for each one of them have IP addressing like subnetting using CIDR method. So it should be considered as of a IP core. Therefore by given descriptions we can define two strategies: 
- Strategy on small sensor of networks with small number of nodes: In these networks we can use single cluster design.

- Strategy on large sensor of networks with large number of nodes: In these networks we can use multi-cluster design.

\section{Cluster Set-uP}

Clustering is a key technique used to extend the lifetime of a sensor network by reducing energy consumption [17]. Now manually we determine these numbers of nodes in sensor field have to be clustered into several groups. Assuming, we need one cluster head for each set of ten nodes, therefore we consider ten clusters, as a result sensor field are divided into 10 sections (clusters).

To identify each node is belong to which cluster, first determine each cluster heads on each cluster. For this purpose as we mentioned on previous section we need ten cluster heads (in fact we are facing large number of cluster heads). Therefore to pick these ten cluster heads, we consider an array length 10, that each section of this arrays belong to each cluster head. In a repeat loop 100 (number of nodes) ten nodes have the highest energies, considered as a cluster head. Then enter the amount of energy on each one of those to sections of arrays. As we mentioned on clustering algorithm, unfortunately with the onslaught of the busy stations the charge capacity at busy stations within a few empty wagons filled. This problem in the metro clustering algorithm could be an idea or advantage for our proposed, it can solve the problem of delay in the recruitment and formation of clusters in wireless sensor networks. In fact each node in network when received a message from cluster head that partially include capacity of cluster, and number of empty space in clusters, should accept the membership of cluster head, and be a member of cluster (of course cluster head also, receives request for membership in cluster that receives, from nodes, and reviews, if there is a empty capacity, it let's them to stay, otherwise prevent nodes from being member of cluster). Now each one of the cluster node should create cluster, and start getting member in its cluster. To specify the cluster range, if we assume each cluster as a circle area with radius $r$, then $r$ will be calculated from following equation 4 .

$$
\mathrm{r}=10 \mathrm{~cm} * \frac{(\mathrm{ECH}) \mathrm{j}}{5 \mathrm{j}} \quad(\text { Equ. } 4)
$$

If a node has the highest amount of battery means $50 \mathrm{j}$ energy, and it is able to send message (broadcast) to $10 \mathrm{~cm}$ radius. Thus with a simple proportion Table $1 \mathrm{we}$ can prove this formula for r. We consider $\mathrm{E}_{\mathrm{ch}}$ as of energy of each cluster head.

Table 1. Value of $\mathrm{R}$

\begin{tabular}{|c|c|}
\hline $5 j$ & $10 \mathrm{~cm}$ \\
\hline$\left(\mathrm{E}_{\mathrm{CH}}\right) \mathrm{j}$ & $(\mathrm{r}) \mathrm{cm}=?$ \\
\hline
\end{tabular}

According to the energies of some cluster heads are the same, and some have different energies. Therefore we can have same size clusters, or different sizes (in fact small, large circles, or equal). And power of sending signals by cluster heads to each one (signals received by regular sensors) for absorbtion, invite nodes decrease with expanding their distance (this rule is taking from \#2 algorithm law of Glow-worms). After determining the area and boundary of each cluster heads, should broadcast their messages as being a cluster head, until the rest of the cluster head aware of it. In fact advertised message with cluster heads contain two subjects: 
1. I am cluster heads, 2. Invite nodes for membership in their own cluster (this idea is taken from algorithm of Glow-worms, Each glow-worm absorb the rest of glow-worm to ward itself by radiation). Operation of sending signals by each one of the cluster head will happen at the same time, and parallel. After each cluster head broadcast its message has become a cluster head to the rest of sensors (sends them a signal), each one of the sensor nodes pick nearest cluster head for themselves. And from the receiving signal energy from cluster heads, and comparing those with each other you find out which one has the higher energy. Then distance of that with cluster head that has higher signal energy, is less, becomes member of that cluster. The routine, continues for rest of the sensor nodes. Until at the end cluster formed, and each cluster head identifies members of it's cluster. After each sensor node picked up its cluster head should its ID number that is unique number send it to its cluster head in sensor field. Cluster head receives this number in a arrays along with other ID's from other nodes and save, store those. Also third level named "alternative cluster nodes" adds to algorithm. This idea is corrected such as, cluster head nodes instead of saving ID of regular nodes should save ID's of "alternative cluster head node". Also "alternative cluster head" should save the ID's of regular nodes for themselves. So when need to be collected all the data take the least delay time inside the network. Therefore reduces the volume and number of same messages that are repeating. One of the problems according to the field, boundary like circle with radius of assumed, some of the clusters are overlapping with each other. Worst than that have node or nodes that are member of two or some clusters. Therefore mentioned node will becomes member of two, or some clusters. But according to this that each of the sensor nodes just should be member of one cluster, and energy of each cluster is different than the other. Therefore this node will becomes member of cluster. That receiving signal from cluster head has higher energy. When the worst thing will happen that this node be a member of clusters which have the same energies, and send signals with equal energies to the mentioned node. Therefore this node get to the 2 way or multi way to pick up cluster.

Getting idea from metro clustering algorithm, it can be considered that: each node for membership between two or some clusters that cluster heads of all are the same (based on sending signal, and receiving by mentioned node) should pick up a cluster that has less member. As a result with this method we have more clusters to be distributed, this way the problem of forming clusters that are lacking of distribution of number of members among them will be solved. Each node after was identified is a cluster, should save on broadcasting messages, number of cluster members in addition two other parameters that mentioned before, on any moment in different variables, and send it to groups. It is known that in the beginning of process, absorbtion of cluster members are equal to 1 .

According to the assumption of proposed cluster algorithm on the beginning of forming itself has at least one member? but who is that member? Answer : cluster head. So, can say each cluster like wagon in metro clustering algorithm has specified capacity. At the beginning of algorithm according to the area covered (sensor field), efficiency of sensor network for special applications, total number of nodes, and also number of clusters, are considered. As a result when the cluster reached to maximum capacity, cluster head send messages to quit broadcasting temporary. When an empty space was created (when the first node dies means when the battery is at the lowest energy) cluster head again act to send messages, broadcast for membership. As we know in some algorithm about clustering, network lifetime depends on the first node dies. Some other believe on lifetime that it is the last node dies. But in our proposed algorithm are given explanation, lifetime of network is the death of last node. Another problem is cluster head accumulate in one area, as a result we will have densed cluster head (close to each other). 
International Journal of Computer Networks \& Communications (IJCNC) Vol.8, No.4, July 2016

Therefore the worst time is when the nodes are far away from cluster heads (more than $10 \mathrm{~cm}$, that one cluster head node with full energy that is considered algorithm 50j). As a result no messages have received from any of the members. Means not going to be a member of any cluster. In this algorithm suppose (we assume) that these nodes are called orphans. So we should change the algorithm some how that cluster head distribute equally along the sensor field to liminate this problem, and cover all of the area. In fact in this condition a important problem is coverage will be discussed. According to the location of each one of the sensor nodes in sensor field it may that one cluster has high number of members, another cluster has less number of member sensor. On the other hand by this method selecting cluster heads, cluster heads may get selected close to each other. In some fields we may face with less cluster head, or even lack of cluster head. In fact can be said method of selecting cluster head in this algorithm is not a good distributed way. We can use second assumption in CICA algorithm that is a kind of colonial competition algorithm, get some ideas. Also use game theories problems. If these nodes have less distance to base station (BS) they are able, instead of waiting for empty location in clusters (or getting messages from clusters for membership), directly send their data achieved from environment, to the base station (BS). Using this idea, also achieving idea from selfish behavior of players (actors) in game theory, we can come up with this result also. In this case clusters that are close to base station, their members (that) have less distance from base station than their cluster head can show selfish behavior, instead of sending sense data to cluster head, send sense data to base station directly. As a result lose less energy by this method, because data will be sent in a shorter distance.

\section{Sleep/Wake Scheduling Algorithm Phase}

As we know one of the cluster heads responsibilities is scheduling. Therefore cluster head can take the nodes to sleep mode, and when those are needed by sending a signal, call them back. But in suggested algorithm, it is assumed, cluster head, instead of putting regular sensor node to sleep mode, can put alternative cluster head to sleep mode. So, when alternative cluster heads were selected, cluster head according to condition of environment and it's cluster, put to sleep mode some of alternative clusters nodes (in fact when the condition of coverage environment are stable). Only one of them are kept as of standby. When it is the end of energy (it's energy is almost finished), Randomly (or selective) one of the alternative cluster head put in a active mode), and by sending message awake, and get ready to work. Using that we have redundancy, fault tolerance among alternative cluster head. We thrift large amount of energy. Cluster heads on sleep mode does not use energy. As a result increases life time of network.

\section{Calculation The amount Of Energy During Sending Data}

The amount of energy consumption during sending data can be calculated by following equation:

$$
E=e(r) * d(i, j)^{b}+e(t)(\text { Equ. 5) }
$$

$E$ is the expenses of two nodes $i, j$ are located with distance $d(i, j)$ from each other. e(r) amount of energy consumption on sending process, and $e(t)$ is amount of energy consumption to send one unit data, one unit distance and $\mathrm{b}$ is the power of lost packets because of channel noise that amount of is between 2 , and 4 based on specific environment and distance between two different nodes. Usually if the distance is less than minimum, $b$ is equall 2, otherwise assumes it 4 . How much we consider the amount of mentioned values above, or energy consumption to receive data, we can use sensors real specific. Amount of unit energy consumption of those to receive, and send one unit we use in a reality in simulation. 
International Journal of Computer Networks \& Communications (IJCNC) Vol.8, No.4, July 2016

\section{Processing Of Intra-Cluster Locally}

Cluster nodes, not only are cluster head, but also like any other cluster head should sense the data from their own environmental. Collect the data from members of cluster node that priority received them from arrays ID. Then, combining them with data collected by itself, process them locally. At last according to the location of it, and distance from base station or BS sent data to that station.

\section{Sending Collected data By Cluster Heads To The Base STATION}

In this algorithm we assume that none of the cluster heads don't send data from their cluster heads directly to the base station. Because algorithm assumes that actually cluster heads act as of backbone in sensor field, and send data to the nearest cluster head around itself. This routine was done to the all cluster heads until at last cluster head that is closer to base station, geographically receives data from other cluster heads. Then combining that with it's cluster data send total data (collect data) to base data.

- Each cluster head, finds the nearest cluster head to itself (nearest neighbor) through routing method.

- Problem of localization are discussed. Each one of cluster heads can find location or distance of itself from a base station by GPS. Using the method waste a lot of energy (sensors that are cluster heads). One of the first (priority) assumptions that sensor nodes, and cluster heads are fixed and stationary.

Therefore localization has to be done only once (through GPS cluster head which has more energy than regular sensors). This cannot be eliminated. But if the assumption is this that each one of clusters are like a square. Therefore we can use grid localization method. In such a way that surface of square should considered grading (a table or 2 dimensions arrays). We consider each one of the cells or rooms of arrays on number with 2 digits that shows row, column of array.

On the other hand area of square figure considers like one two dimension array over $\mathrm{x}$-axis on positive direction. Example (Figure 1). By this method, X-axis of each one of the cluster nodes will be identified. Also BS in a square shape area are larger than sensor field, using this method we determine the X-axis, and then compare with each cluster nodes. At end the cluster head that has least distance from 2 dimensions $\mathrm{x}$, $\mathrm{y}$ with BS, receives the data from the rest of cluster head, and send to BS. On a condition that sensor field be circle shape, for localization of sensor nodes environment sensor field we can consider similar circle geometry rational. Location of each node in this circle using formulas geometry rational are calculated. Also for triangle sensor fields we can use localization Pythagorean method of positioning. Therefore localization has told one should only once localization (through GPS cluster head which has more energy than regular sensors was done. We can convert any other geometric figure to a combination of these three figures. For example lozenge can be divided into two triangles, trapezoid can be divided into a square, and a triangle, rectangular can be divided into two square or without converting to square, taking directly to $\mathrm{X}$-axis. 


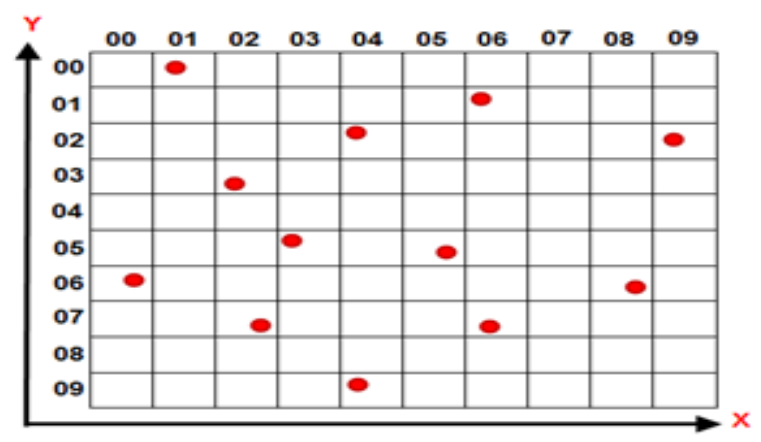

Figure 1. Shows location analysing, of grid

\section{Sending Collected Data By Cluster Heads To Base Station In A Multi-HOP Way And Co-Operation}

Cluster heads are in mutual with each other (according to assumption backbone of cluster heads). Therefore we can say in this algorithm relationship inter-cluster will be type of multi-HOP. Because it may be transferred data from one cluster to the different cluster heads, at last reach to the base station. Using this method not only reduces the overhead cluster heads. But also increases the load balancing among cluster heads. Cluster heads will co-operate with each other send data to base station. Similar to MPLS network it is made of backbone that uses sensors of cluster heads. In fact cluster heads play the role of backbone routers in wireless sensor in network. In this condition we can consider as many as BS, instead of one BS, according to application of sensor of network. All BS are located in a environment outside of super network (this idea according to sensor of networks, we consider that BS always are in a area very far away from the cluster. With this assumption, each cluster head in super sensor networks that are closer to considered BS, should collect the data from other cluster heads then collect, and combine them locally. Super sensor networks on previous stay are include cluster head. In fact is made of cloud from clouds, or clusters, and we can say sub sensor cloud. Finally process the data, and send them to the closest cluster head which has the least distance from it (Figure 2).

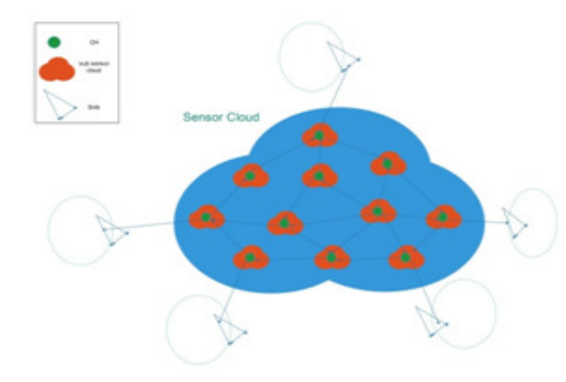

Figure 2. Multi-hop in multi-BS networks

Each one of the cluster head should send a signal to each. One of the (BS'S) base station, and wait to get signals from BS's. At least comparing energy signal return (every signal have energy). Each cluster head has received higher return energy is considered as gateway or exist point wireless sensor network (Figure 3). 


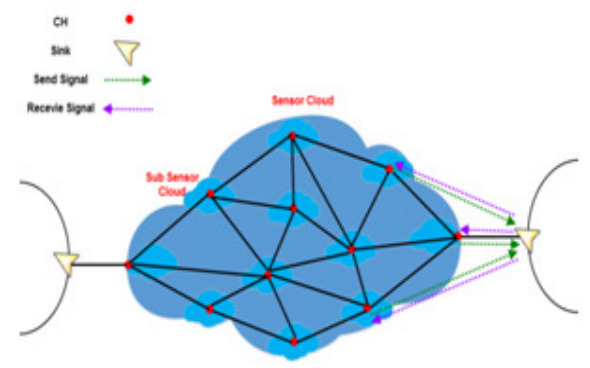

Figure 3. Shows gateway in Multi-BS networks

This method are used for expanding of sensor networks. Therefore BS of one network transfer data to another BS of sensor network through cluster head of backbone. Cluster head that has the shortest distance until sender BS, ingress $\mathrm{CH}$, and cluster head that has the shortest distance until receiver BS, are called egress $\mathrm{CH}$. In general (each one of them) or both of them are called edge $\mathrm{CH}$. Also other cluster heads are called backbone $\mathrm{CH}$ (Figure 4).

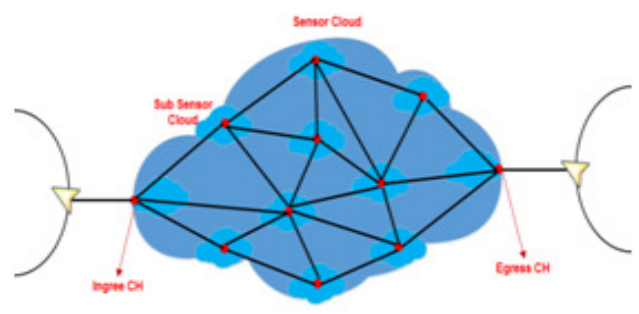

Figure 4. Cloud network in Multi-BS networks

In Multi-BS networks situation of sinks are unknown eventhough they are fix and stationary. Cloud sensor network will solve problems of Multi-BS. In mobile Multi-BS networks of sinks always moving, and have mobility around sensor field. Also solves problem of mobile Multi-sink. Because every time one of the cluster head nodes find less distance toward considered sink, and act to accumulate data from the rest of cluster head, will to refer sink. (like animal husbandry network field or know project zebranet).

If CH's could encapsulate data using one of data compact method. As a result less energy are used to send compact data (volum decrease) to the base station. This matter cause reduce of energy consumption, as a result will increase life time of sensor nodes, and of course life time of network.

Another benefit of other sensor cluster heads are, when all of sensors of one cluster feel the same sense. First they send to alternative cluster head nodes. They also do necessary processing, and only send one message to the cluster head (for example: message "fire" when there is a fire in field). Cluster head also process less received messages, and can use it's energy to send, or encapsulation data. As a result life time of network will increase. Probably the problem of this method, be the processing of received messages to alternative cluster nodes that uses their energies. But on the other hand existance of these nodes reduces the overlapping process from cluster head nodes reduces very much. So processing speed will increase and have less delays. As a result by doing compacting, cluster heads use less of their energies, as a result life time of network increase. One one the simplest uses encapsulating algorithm means encapsulation. 
Therefore each cluster head before sending cluster's data to another cluster head or BS, adds one header or one trailer to it's main data contain or sending packet. Then tend to send (same as encapsulation method packets on reference model OSI or VPN protocols.

Now, when packet receives to next cluster head. First cluster head, header, and trailer takes a packet from it.

Means decapsulate it. Cluster data append or add to the end of it. At last before sending to next cluster head or base station, header, and trailer will be added to it. Finally, total packet that is collected data from all of the clusters will be sent to base station. BS also does decapsulate, and final data will be deriven from it. It is obvious that operation of encapsulation and decapsulation will take very little energy from cluster head. But the main advantage of it, will be decrease of volum of packets, and as a result consumption of energy, and increase lift time of network (Figure $5)$.

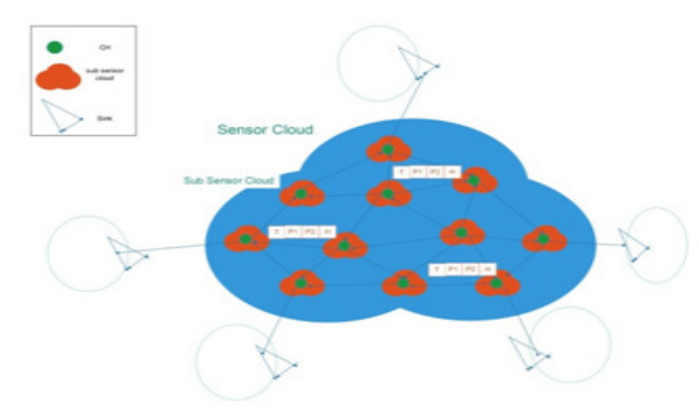

Figure 5. Encapsulation, and decapsulation in Multi-BS networks.

\section{Using Sensor Backbone Network InClude Cluster Head, And Solving The Problems Of Multi-BS Network}

Also using backbone method can solve problems of Multi-Bs networks. For this purpose we use one backbone network or sensors cluster heads. In fact cluster heads will play the role of backbone routers. In this condition we can consider as many as BS is necessary instead of one BS. These are located outside of cloud sensor network environment (This idea according to in sensor network of BS always is farther from cluster it come to my mind). With it assumption, each cluster head in cloud sensor network that is closer to BS, should collect the data from other cluster heads. After combining and processing those locally (assume cloud sensor network in previous stage itself include clusters. In fact cloud made of clouds or clusters were made, we can call clusters, sub sensor cloud) process, and send them to the next cluster head that have the least distance away from them (Figure 6). 


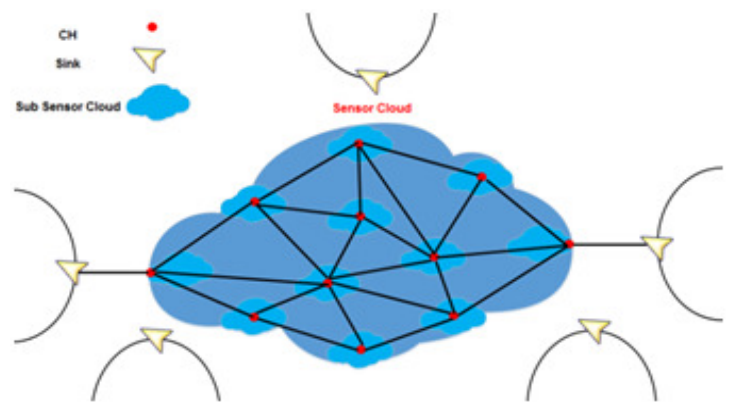

Figure 6. Backbone in multi-BS networks.

\section{Using 3-Level Hierarchical Clustered Heterogeneous WSN ANd USE Of Social INTELLigence, INTRA-CluSter Mutual GrouP}

It may all the member of one cluster sense all the natural happening know around themselves or sense( For example increase in temperature in fields when firing, increase in humidity, or Gas leaking in pips,...) equal sense from environmental. All the members of cluster send these equal data to cluster head. Cluster head also should process locally all the received data, before sending to BS. Since equal data receives from all the members, then waste a lot of time, and energy from cluster head. Therefore, for delay on process, decrease expense, energy consumption for process, decrease numbers of received messages, sending intra-cluster I decided two levels sensors that in all articles, and references are used, divide those to 3 different levels of energies. This idea according to colony structure algorithm of bees, similarity of that with structure of hierarchical wireless heterogeneous sensor networks is achieved. Actually like bees colony algorithm that considered 3-levels of bees: Queen, Male bees, and labor bees. On new sensor leveling, we consider three different levels as a regular nodes are assumed, number of them that have more energy, and the next period, may become cluster head. As a alternative cluster head nodes (as a male bees) will be selected in cluster head. Finally remaining nodes that have the lowest, least level of energy, as regular nodes (as a labor bees) will be selected. As in this comparison we can say each cluster will be considered as a colony. As we assumed on colony of bees that a bee that has the most energy will be selected as a queen. Therefore it is necessary existence of one cluster head on each cluster (Figure 7).

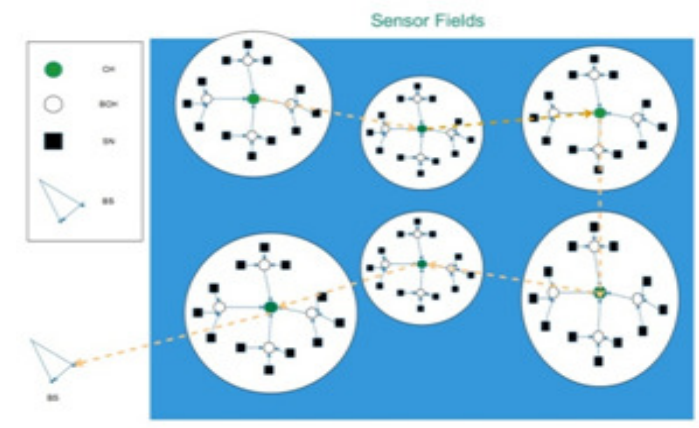

Figure 7. Tree levels sensor network

If we assume that connection intra-cluster is from Multi-HOP kind. Also regular sensor nodes on each cluster have mutual relationship, with alternative cluster head nodes. Also alternative cluster head with cluster head node have mutual relationship. We can reach to a quite tree structure. This 
structure include one root node that is called cluster head. Alternative cluster head nodes, and finally reach to a leave nodes or regular sensors. We can say that about rest of the cluster, and also on total sensor field. Finally we get to the point-to-point connection between the last cluster head and BS. But the the assumption is that in one cluster there is not a single connection singleHOP, each regular sensor or MS should be on connection by alternative cluster head node with selected cluster head.

\section{Using Group Alternative Cluster Head Nodes For Selecting Cluster Head On Each Round}

We assume in this algorithm on each round, cluster head was selected among the rest of nodes in clusters, that has the most remaining energy. Therefore with respect to this assumption of alternative cluster head on each cluster (as of HEAD algorithm, that is in reality equal to male bees in colony of bees) that takes less time on selecting cluster heads on next round. This matter decrease the selection of cluster head, and on the hand because we have less nodes to select, therefore use less energy. As a result lifetime of network increase.

\section{Simulation, And Analysis Of Parameters Of Service Quality}

The analysis of results reveals how the idea to the realization joins. In this section, we present our simulation setup for 802.15.4, LEACH, CBRP, and proposed method protocol, followed by the performance metrics and comparisons. The main goal of simulation is to analyze the performance of different routing. We choose to simulate the NS 2 simulator to make it more flexible and has better performance.

In this section we simulate and implement the three protocols and proposed method. Simulation was performed with the software simulator NS 2.34, NS 2.29 on Linux. Values of the simulation parameters are given in Table 2.

Table 2. Values of Parameters for simulation

\begin{tabular}{|c|c|}
\hline Parameters & Values \\
\hline Simulator & NS 2.29 and NS 2.34 \\
\hline Routing & AODV \\
\hline Simulation duration $(\mathrm{Sec})$ & $10,60,80,100,120,140$ \\
\hline Simulation area & Ch $1000 \mathrm{~m}$ \\
\hline Number of nodes & Chain topology \\
\hline Transmission range & 200 \\
\hline Movement & 4 packets/sec \\
\hline MAC Layer & CBR (UDP) \\
\hline Queue Size & 512 bytes/packet \\
\hline Packet rate & LEACH, 802.4.15, CBRP, Proposed method \\
\hline Traffic type & \\
\hline Data payload &
\end{tabular}

Simulation time is 140 second. We achieve values of parameters of QoS at 40's up to 140's degrees, and then compare those with each other. These values are calculated using C++ codes. The simulation scenario is obtained and discussed according to the simulation settings (Table 2). In the simulation environment, the sensor was considered $1000 \mathrm{~m} * 1000 \mathrm{~m}$. Assume that the 
range of the sensor is 20 meters. In addition the initial energy of each sensor node was chosen $0 \mathrm{j}$ up to $50 \mathrm{j}$. Test for the number of sensor $\mathrm{N}$ nodes were performed, was equal to 200 . Then, using scenarios, we analyze separately the parameters of QoS. The results obtained were compared with proposed method.

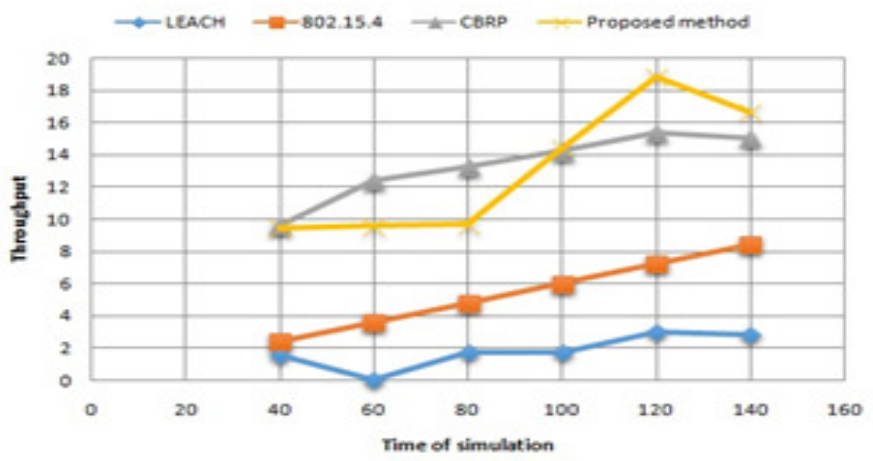

Figure 8. Throughput comparison

The effective number of data current at a certain time of transfer are called throughput. This mean to total of packets received at the destination in a node is called throughput. These parameters are used as the band width in some situations. In general, larger throughput network, performance is better. Figure 8 shows the amount of protocols throughput the time of 140 seconds. As you can see proposed algorithm shows more throughput . As a result performance of proposed method is better than other methods.

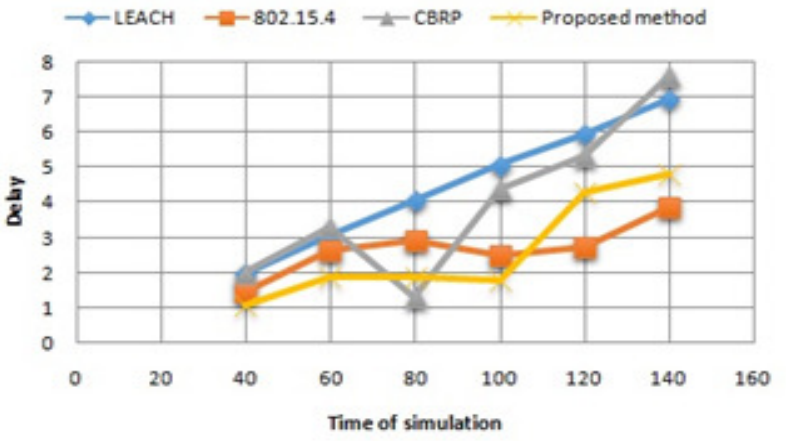

Figure 9. Comparison of delay time on sending packets.

The delay metric between two nodes represented as is the sum of the processing, queuing, transmission, and propagation delay [6]. The average data delivery delay is the end-to-end delay experienced by successfully received packets [13].

Figure 9 shows the amount of delay time on received packets at the time 140 seconds. As it is identified received packets shows less delay time using proposed algorithm method with respect to other algorithm. Cause of this decrease can say, delay on process of membership of clusters, and as a result a little delay on construction of cluster. On proposed clusters algorithms coverage by regular nodes for membership or none membership in clusters and selection of cluster head can be done by quick decision, and little delay. Also by processing intra-cluster locally, using alternative cluster head nodes reduces delay time. 


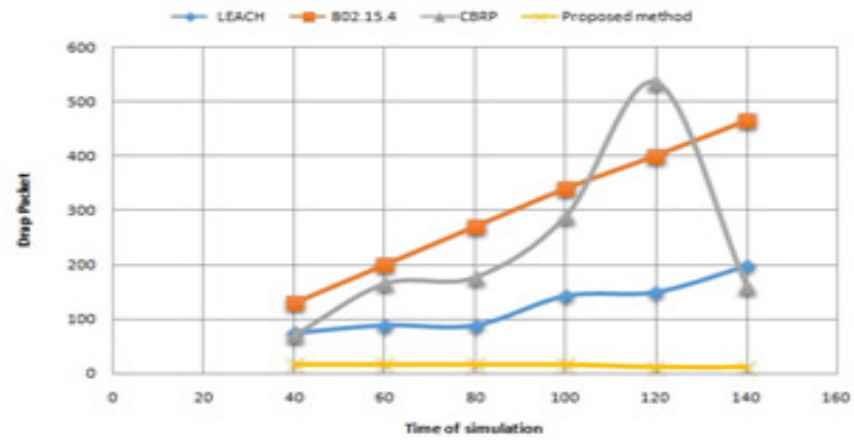

Figure 10. Comparison of lost packets

The simulation is carried out with 200 active nodes. One of them being the coordinator. The nodes remain stationary. Figure 10 number of lost packets when connection is going to be done among source node and destination are shown at the time of 140 seconds. As you have seen on proposed algorithm amount of lost packets are very low. The reason for this decrease, increase of processing speed intra-cluster by decrease of load or overheating of process and dividing the load (or load balancing) between cluster head, and alternative cluster head nodes.

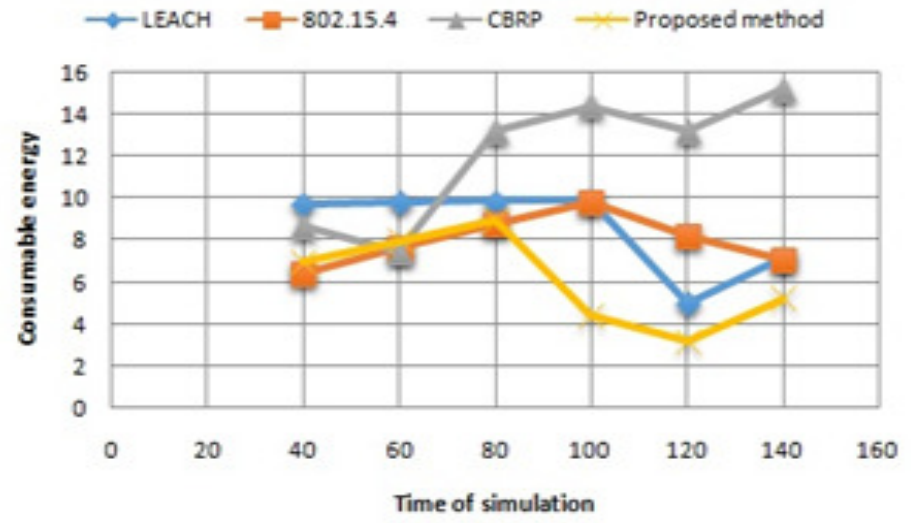

Figure 11. Comparsion of consumption energy

As, a certain number of nodes are selected to transmit results to the gateway, the network might consume energy differently depending on the network topology and the number of information transmitting nodes. The average energy consumed is an indication of the energy consumption in transmission and reception of all packets in the network. This metrics reveals the efficiency of an approach with respect to the lifetime of a WSNs [13]. The average energy consumption per transmission is the index of the network lifetime; less energy consumption per transmission indicates more network lifetime. Network lifetime is given in terms of when the energy of a first node drops under the energy threshold [14]. Amount of energy consumption on total network nodes are shown in Figure 11. As it is obvious in picture energy consumption of proposed algorithm with respect to 3 other algorithm are very low. The reason for this decrease is, increase in speed of cluster head during next rounds, decrease on volume of number of repeating (same) sending messages intra-cluster by alternative sensor cluster nodes (using accumulate intelligent). 


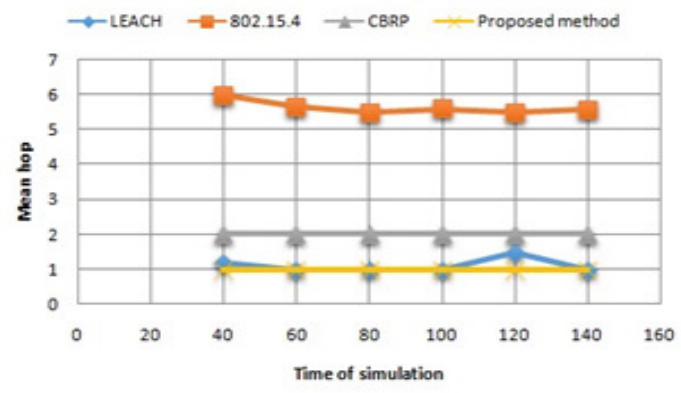

Figure 12. Comparison of average HOP

Figure 12 shows number of steps receive the packet to the destination the 14 seconds. As it is shown on figure the packets receive to destination using proposed algorithm used less number of steps that other algorithms. The main reason of decrease, decrease on connection of inter-clusters, and intra-clusters (number of routes to transfer data) is using Multi-HOP. Also using method to find the shortest distance between neighbors by ants colony routing algorithm.

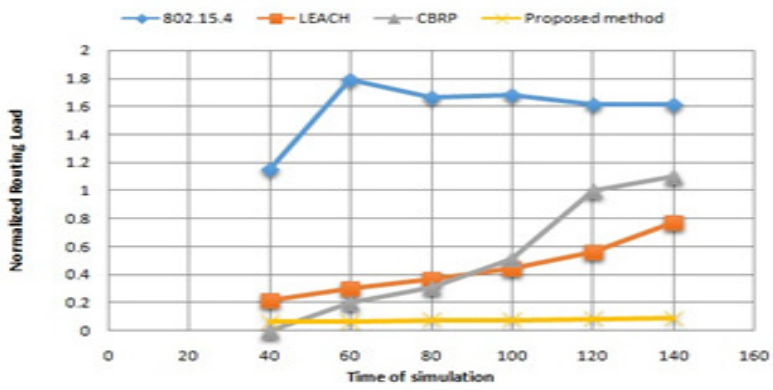

Figure 13. Comparison of jump (traffic or network load)

Figure 13 number of jumps (traffic or network load) of traffic at the time of 14 seconds (during the 14 seconds). As it is shown in picture using proposed traffic algorithm, network load is less. The reason for this decrease, decrease of volume of number of send repeating transfer (same) intra-cluster is by alternative cluster head sensor (using accumulate intelligent). Also it is divided load between cluster head nodes, and alternative cluster head nodes.

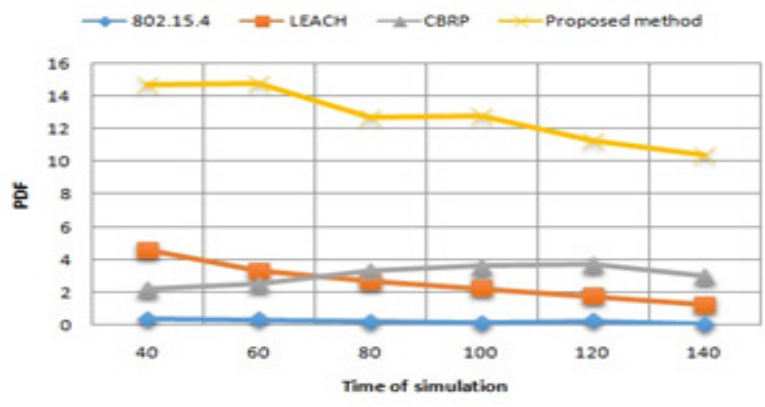

Figure 14. Comparison received packets rate 
The packet delivery ratio is one of the most important metrics in real-time applications which indicates the number of packets that could meet the specified QoS level [13]. It is the ratio of successful packet receptions referred to, as received packets, to the attempted packet transmissions referred to as sent packets [13]. Whatever the rate of delivery of packets be higher, performance of the network is good, and it means the performance of protocol in the network is better, if it is low, then the performance is not good. Number of received packets in network is depends on average of lift time sensors. Figure 14 rate of delivery of packets to destination at the time of connection between source nodes and destination at the time of 140 seconds is shown. As it is shown on the figure with proposed algorithm amount of received packets to destination at the time of connection between source nodes and destination are very much. Therefore average lifetime of sensors are higher.

\section{Compared The Proposed Algorithm With 10 Clustering Algorithms In The AREA OF Wireless SenSOR NeTWORKS}

\subsection{To compare the parameters that were considered. Include: (Table 3)}

- Select a node in the cluster process parameters.

- Distributed algorithm is centralized or hybrid.

- The distance from the head to the base station.

- CH distributed. The amount of overhead in the selection process of cluster heads.

- Taking into account the residual energy of cluster heads selection process. The delay in the selection process cluster.

\subsection{The parameters that influence in the form of cluster nodes without a cluster}

- Distance from cluster node cluster node without

- Without a cluster head node to node distance clustered.

- Cluster size

- The number of steps (1: a single step, 2: the two-step, k: the k- step) (In fact, more than one step is called a multi-step)

- Overhead recruitment process non-cluster nodes in a cluster.

Table 3. Summary comparison clustering algorithms.

\begin{tabular}{|c|c|c|c|c|c|c|c|c|c|c|}
\hline \multirow[b]{2}{*}{ Scheme } & \multicolumn{6}{|c|}{ Cluster Heed(CH) Selection } & \multicolumn{4}{|c|}{ Cluster Formation for non-CH nodes } \\
\hline & $\begin{array}{c}\text { Distributed } \\
\text { centralized } \\
\text { hybrid }\end{array}$ & $\begin{array}{l}\text { Distance } \\
\text { from } \mathrm{CH} \\
\text { to the BS }\end{array}$ & $\begin{array}{c}\mathrm{CH} \\
\text { Uniform } \\
\text { Distribution }\end{array}$ & $\begin{array}{l}\text { Overhead } \\
\text { In } \mathrm{CH} \\
\text { Selection }\end{array}$ & $\begin{array}{l}\text { Residual } \\
\text { Energy }\end{array}$ & Delay & $\begin{array}{l}\text { Distance } \\
\text { Non-CH } \\
\text { To } \mathrm{CH}\end{array}$ & $\begin{array}{c}\text { Cluster } \\
\text { Size }\end{array}$ & Hoops & $\begin{array}{c}\text { Overhead } \\
\text { to a } \\
\text { Cluster }\end{array}$ \\
\hline LEACH & Distributed & No & No & Low & No & Low & Yes & No & T & Low \\
\hline LEACHC & Conteralized. & Yes & Yes & High & Tes & High & Yes & Yes & $T$ & $\mathrm{High}$ \\
\hline $\begin{array}{c}\text { TL- } \\
\text { LEACH }\end{array}$ & Distributed & No & No & Medium & No & Med & Yes & No & 2 & High \\
\hline HEED & Distributed & No & Yes & High & Yes & High & No & Tes & 1 & Low \\
\hline EECS & Distributed & Tes & Yes & Low & Yes & Low & Tes & Yes & $T$ & Low \\
\hline EEHC & Distributed & No & No & Low & No & Low & Yes & No & $\mathrm{k}$ & High \\
\hline MRPUC & Distributed & Yes & Tas & Low & Tes & Low & Tes & No & 1 & Low \\
\hline PEACH & Distributed & No & No & Low & No & Med & Yes & No & $\mathrm{k}$ & Low \\
\hline S-WEB & Hybrid & Yes & Yes & Low & Yes & Med & Yes & Yes & 1 & Low \\
\hline EEUC & Distributed & Yes & Yes & Low & Yes & Low & Yes & Yes & $\mathrm{k}$ & Low \\
\hline LEH3LA & Hybrid & $?$ & No & Low & Yes & Low & $?$ & $?$ & 2 & Low \\
\hline
\end{tabular}

\section{CONCLUSION}

In this article a leach extended hierarchical 3-level (LEH3L) heterogeneous clustering algorithm, and dynamic to improve QoS, and lifetime in wireless WSN are presented. In this protocol 
selecting auction cluster head like simple 2 steps. Connect intra-clusters, and inter-clusters implemented such a Multi-HOP. Also according to this algorithm a structure of 3-level hierarchical clustered heterogeneous are presented, that obey from the colony of bees algorithm include: cluster head nodes, alternative cluster head nodes, ordinary nodes. This algorithm operates based on remain energy, and ID of selecting cluster heads on each round. It has the ability of encapsulation of data by cluster heads. Some how uses the simplest method of encapsulation. Data security by cluster heads increases. This protocol of Multi-BS or Multi-sink and has ability to solve the developing wireless sensor networks, it has the ability of development of using backbone network, organized of cluster heads. Volume of number of repeat (same) sending intra-cluster by alternative cluster head sensors (using accumulate intelligent) will decrease. And also lifetime of wireless sensor networks consumption of sensors energies, will increase. Sleep mode on alternative nodes in clusters instead of ordinary sensors was used. Redundancy, fault tolerance between alternative cluster heads was increased. Delay time intracluster locally using alternative cluster head node have decreased. Processing speed of intracluster by load decrease or process overheating and load balancing between cluster head node and alternative cluster heads was increased. This protocol causes improvement parameters of service quality on protocols 802.15.4, LEACH, and CBRP.

\section{REFERENCES}

[1] Akyildiz I. Su W. Sankarasubramaniam Y. and Cayirci E., (2002) "A Survey on Sensor Networks, "IEEE Communications Magazine, 40,102114. http://dx.doi.org/10.1109/MCOM.2002.1024422

[2] Brienza S., (2013) "Strategies for Optimal MAC Parameter Setting in IEEE 802.15.4 WSNs: A Performance Comparison, Computers and Communications(ISCC)," IEEE Symposium on Computers and communications (ISCC), Split, 000898-000903.

[3] Lotf J.J., (2010) "Quality of Service in Wireless Sensor Networks," The Third International Conference "Problems of Cybernetics and Informatics", Baku, 6-8 September.

[4] Chen D. and Varshney P., 92004) “QoS Support in Wireless Sensor Network: A Survey,” Proceeding of International Conference on Wireless Networks (ICWN 04), Las Vegas, 21-24, 227-233.

[5] Barai L. and Gaikwad M., (2014) "Performance Evaluation of LEACH Protocol for Wireless Sensor Network," International Journal of Innovative Research in Advanced Engineering . (IJIRAE), 1, 141 145. http://ijirae.com

[6] Mohammadi Baygi S.J. and Mokhtari M., (2014) "Evaluation Performance of Protocols LEACH, 802.15.4 and CBRP, Using Analysis of QoS in WSNs," Wireless Sensor Network, 2014, 6, 221-236, Published Online October 2014 in SciRes, 2014. http://www.scirp.org/journal/wsn ,http://dx.doi.org/10.4236/wsn.2014.610022

[7] Huang X. and Fang Y., (2008) "Multiconstrained QoS Multipath Routing in Wireless Sensor Networks," Wireless Networks, 14, 465-478. http://dx.doi.org/10.1007/s11276-006-0731-9

[8] Radi D., (2008) "Favor of Improving Quality of Service in Wireless Sensor Thunderstruck Using Multiple Routing," Quarterly-Scientific Research Chamber of Electrical Engineering.

[9] Hosseini-Seno S.A. and Wan T.C. and Budiarto R., (2009) "Energy Efficient Cluster Based Routing Protocol for MA-NETs," Proceeding of International Conference on Telecom Technology and Application, Manila, 6-8, June 2009.

[10] Ali K.A. and Mouftah H.T., (2001) "Wireless Personal Area Networks Architecture and Protocols for Multimedia Applications," Ad Hoc Networks, 9, 675-685. http://www.elsevier.com/locate/adhoc

[11] Mbowe J.E. and Oreku G.S., (2014) "Quality of Service in Wireless Sensor Networks," Wireless Sensor Network, 6, 1926,2014.http://dx.doi.org/10.4236/wsn.2014.62003235

[12] Rahmani E., (2005) "Design and Modelling of the Physical Layer Standard of IEEE 802.15.4. Panzdhymn Iranian Conference on Electrical Engineering," Iran Telecommunication Research Center, Tehran, 2005.

[13] Meybodi M., (2009) "Quality Control Services Based on Wireless Sensor Networks Energy Sensor," 15th Annual International Conference of Computer Society of Iran, Tehran, 2009. 
[14] Priyanka S. and Kumar M. and Jaiswal A. and Saxena R., (2013) "Analysis of Zig-Bee (IEEE 802.15.4 Standard) for Star Topology with AODV Protocol," International Journal of Innovative Technology and Exploring Engineering (IJTTEE).

[15] Kauar T. and Paulus R. and Jaiswal A.K. and Ashok A., (2014) "QoS Analysis in IEEE 802.15.4 and Impact of Flag Mobility on Network Performance in WSN," International Journal of Computer Applications,90,16-20.

[16] Kumar1 V.N. and Sankar K.S. and Srinivasa Rao L. and Santhi Prabha I., (2012) "Comparative Analysis of QoS-Aware Routing Protocols for Wireless Sensor Networks," Innovative Systems Design and Engineering.

[17] Katiyar V. and Chand N. and Soni S., (2010) "Clusterning Algorithm for Heterogeneous Wireless Sensor Netwok: A Survey," International journal of applied engineering research, dindigul Volum 1.

[18] Afsharzadeh Kazerooni A. and jelodar H. and Aramideh j., (2015) "Leach and HEED Clustering Algorithm in Wireless Sensor Networks: A Qualitive Study,” Advances in Science and Technology Research Journal Volume 9, No. 25, March 2015, pages 7-11,DOI: 10.12913/22998624/1918

[19] Javaid N. and Babar Rasheed M. and Imran M. et al ., (2015) "An energy-efficient distributed clustering algorithm for heterogeneous WSNs," EURASIP Journal on Wireless communications and Networking (2015) 2015:151, DOI 10.1186/s13638-015-0376-4

[20] Sangeetha C P, Dr. C.D. Suriyakala, (2013) “The IEEE 802.15.4 MAC Unreliability Problem: Issues and Solutions-A Survey," nternational Journal of Advanced Research in Electrical, Electronics and Instrumentation Engineering.

\section{AuTHORS}

Mehran Mokhtari : Programming language C, C ++, Pascal, VB, C \#, HTML database SQL, Oracle and Linux Oracle Windows operating system, Simulation with NS-2.26 to NS-2.34 (Fiber, Wireless, WSNs, mobile, etc.), Colored Petri nets CPN, Simulation and modeling CPN Tools, IT (Information Technology), Information and Communication Technology ICT (Information Communication Technology),TRIZ, Project management (management activities, project planning, project scheduling, risk management, etc.), Project management (risk management, ...), Parallel programming,

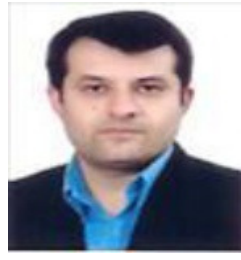
MPI, OPEN MP, Thread, Pthread ,Cloud Computing cloud computing or cloud computing, Mobile Ad-hoc Network, Sensor Networks Sensor Networks, Network Protocols network protocols, multiple routing algorithms, OSPF, RIP, TCP, UDP, Hierarchical clustering algorithms CBRP, 802.15.4, LEACH, Quality of service in a virtual private network (VPN) and wireless sensor networks, http://sama-gh.com/asatid/post556.html

Masoumeh Tavakoli :Teacher of Education Ghaemshahr,IRAN. Fields of interest: ICT, E-learning.

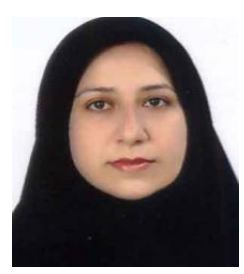

